\title{
Breakdown voltage and triggering probability of SiPM from IV curves at different temperatures
}

\author{
N. Dinu ${ }^{\mathrm{a}, \mathrm{b}}$, A. Nagai ${ }^{\mathrm{c}, *}$, A. Para ${ }^{\mathrm{d}}$ \\ ${ }^{a}$ was with the Laboratory of Linear Accelerator $\&$ University Paris 11, CNRS / IN2P3, \\ 91898 Orsay Cedex, France \\ ${ }^{b}$ she is now with ARTEMIS laboratory, CNRS/INSIS, UCA, OCA, F-06304, Nice Cedex, \\ France \\ ${ }^{c}$ is with the Laboratory of Linear Accelerator 8 University Paris 11, CNRS / IN2P3, 91898 \\ Orsay Cedex, France \\ ${ }^{d}$ is with the Fermi National Accelerator Laboratory, Batavia Il, 60510-5011 USA
}

\begin{abstract}
This work presents a physical model describing the IV curves of SiPM detectors allowing to easily determine important device parameters like breakdown voltage $V_{B D}$ and the shape of Geiger triggering probability $P_{G e i g e r}$. We measured IV curves and tested our IV model in a temperature range $-35^{0} C<T<$ $+35^{0} \mathrm{C}$ on various SiPMs from two vendors (Hamamatsu devices of $3 \times 3 \mathrm{~mm}^{2}$ total area and $50 \times 50 \mu m^{2} \mu$ cell size, 2011 and 2015 year production runs and KETEK devices of $0.5 \times 0.5 \mathrm{~mm}^{2}$ total area and $50 \times 50 \mu \mathrm{m}^{2} \mu$ cell size, 2015 production run). The shape of IV curve can be described in terms of Geiger probability and afterpulsing in a very large current range of $10^{-12} \mathrm{~A}<$ $I_{\text {post }-B D}<10^{-5} A$ over the full working range of all tested devices. It has been also found that $V_{B D}$ from IV curves is slightly higher (few hundred $\mathrm{mV}$ ) than the "breakdown voltage" determined from the usual method of linear fit of gain as a function of bias voltage, this discrepancy reflecting the fundamental difference in the physical significance of the "breakdown voltage" determined by these two methods. The recent generation of SiPMs have very wide working range and there is an evidence phenomena beyond afterpulsing like heating or non-quenched pulses contributing to the fast increase of the current at high bias
\end{abstract}

\footnotetext{
* Corresponding author

Email address: nagai@lal.in2p3.fr (A. Nagai )
}

Preprint submitted to Journal of LATEX Templates

May 26, 2016

(C) 2016. This manuscript version is made available under the Elsevier user license http://www.elsevier.com/open-access/userlicense/1.0/ 
voltages.

Keywords: Silicon PhotoMultiplier, Geiger triggering probability, breakdown voltage, IV model

2010 MSC: 00-01, 99-00

\section{Introduction}

Silicon PhotoMultiplier (SiPM) detector has become an alternative photon detector for many applications that would employ typically PMTs or MA-PMT. Its important advantages such as compactness, speed of response, insensitivity

5 to magnetic fields, high gain, and low operating voltage, make it very attractive in medical imaging applications for diagnostic and therapeutic purposes [1, 2] as well as for high energy physics [3] or astrophysics experiments [4, 5]. To achieve the best performance for a given application, the important device parameters related to avalanche multiplication such as breakdown voltage $V_{B D}$ and triggering probability $P_{\text {Geiger }}$ have to be known. Usually these parameters are determined from dynamic-type measurements (so-called $\mathrm{AC}$ ) which require quite complicated data acquisition system (i.e. amplifier, oscilloscope), long data taking time and precise analyzing procedure of SiPM pulses [6, 7,. Use of static-type measurements (so-called DC) would significantly simplify the analysis procedure 15 particularly for large scale production, since it requires only current-voltage IV measurements with simple connection of the device to a source-measure instrument. The inverse logarithmic derivative method is often used to determine $V_{B D}$ from IV curves [8]. This method postulates that the breakdown voltage corresponds to the maximum of the inverse logarithmic derivative of a current.

20 It is lacking physical motivation and the resulting breakdown voltage depends on the relative magnitude of the avalanche-induced current and the parasitic currents [9]. In this paper, we present a physical model describing the shape of the IV curves over the full working range of various SiPMs in terms of a linearly rising current due to free carriers modified by the Geiger probability ${ }_{25} \quad P_{\text {Geiger }}$ near the breakdown and the afterpulsing effects at higher bias volatges. 
A comparison of detectors characteristics like $V_{B D}$ and $P_{\text {Geiger }}$ calculated using our IV model with those determined from AC measurements is also given.

\section{SiPM samples and experimental set-up}

We have examined three Hamamatsu MPPC detectors (called hereafter, for

30 simplicity, HPK SiPM) of $3 \times 3 \mathrm{~mm}^{2}$ total area and $50 \times 50 \mu \mathrm{m}^{2} \mu$ cell size (S10931050P - 2011 year production run; S13082-050CS(X) and S13360-3050CS, 2015 year production run) and two KETEK SiPM devices of $0.5 \times 0.5 \mathrm{~mm}^{2}$ total area and $50 \times 50 \mu m^{2} \mu$ cell size (SiPM19-W3-MP50NT - no trenches; SiPM19-W3MP50-V1 with trenches, both devices of 2015 year production run). Based on the vendors data sheets, both HPK and KETEK SiPMs are optimized for blue light detection (i.e. $p^{+} / n$ junction implanted in an n-type epitaxial layer grown on n-type silicon substrate).

The SiPM sample under study is measured in the temperature range $-35^{0} \mathrm{C}<$ $T<+35^{0} \mathrm{C}$ of $10^{0} \mathrm{C}$ step using a closed-cycle He cryocooler working on the Gif40 ford McMahon thermodynamic principle (i.e. compressor, cold end, gas lines) and a vacuum chamber at $P<10^{-4}$ mbar.

A copper disk, in tight contact with the cold end, was used to attach simultaneously four SiPM samples and temperature sensors. One temperature sensor, close to the SiPM under test, was used as reference, while the others were used to control the uniformity of temperature over the full surface of the $\mathrm{Cu}$ disk.

The reference temperature sensor together with a heater $(50 \Omega)$ allowed to control and stabilize the temperature to the desired value using the PID system of a LakeShore 335 temperature controller. Each measurement is carried out after waiting for stabilization of temperature (typically half an hour), until the reference sensor reports the temperature to be within $0.1^{0} \mathrm{C}$ with respect to the desired value.

Using the described set-up, both DC and AC tests were performed on our SiPMs samples. During DC measurements, the SiPM sample was directly con- 
nected to a Keithley 2611 source-meter for bias voltage supply and current measurement and the IV characteristics were acquired in dark conditions at various temperatures.

During AC measurements, the SiPM sample is connected to a Miteq AU1332 amplifier (gain $=55 \mathrm{~dB}$, bandwidth $=500 \mathrm{MHz}$ ) while the data acquisition

60 (i.e. waveforms) is provided by a Tektronix TDS5054 oscilloscope (bandwidth of $20 \mathrm{MHz}$ was used for noise suppression). The readout electronics was located outside the vacuum chamber, at room temperature, to provide the same experimental conditions (amplifier gain, electronic noise level) independent of temperature. A Pilas diode laser of $635 \mathrm{~nm}$ wavelength has been employed to illuminate the surface of the SiPM with low intensity light pulses (few photons/pulse) via an optical fiber entering the vacuum chamber.

\section{Dynamic AC acquisitions / waveform analysis}

The breakdown voltage, $V_{B D}$, and $P_{\text {Geiger }}$ have been firstly determined by analyzing the waveforms acquired using AC set-up. At each temperature 10000

70 waveforms of $2 \mu s$ length were acquired at different bias voltages $V_{\text {bias }}$. Data acquisition was triggered by the laser controller, the laser signal being positioned at $1.55 \mu \mathrm{s}$ after the beginning of the waveform. The breakdown voltage of SiPM changes with temperature [8, 10] and therefore, the range of bias voltages, $V_{\text {bias }}$, corresponding to each SiPM sample was selected to correspond to the same overvoltage $\left(\Delta V=V_{\text {bias }}-V_{B D}\right)$ for all temperatures. The HPK SiPM sample from 2011 was measured in a $\Delta V$ range of $0.4 V<\Delta V<2.7 V$, while both HPK and KETEK SiPM samples from 2015 were measured in a $\Delta V$ range of $0.4 V<\Delta V<10 \mathrm{~V}$. The upper limit of the bias voltage range is imposed by onset of strong afterpulsing leading to the amplifier saturation and baseline so $\quad$ shifts. The Hamamatsu devices from 2011 have a working $\Delta V$ range of only 2.7 $\mathrm{V}$ while the latest generation of both vendors have a larger $\Delta V \sim 10 \mathrm{~V}$, showing a considerable improvement of SiPM technology.

Single SiPM pulses (i.e. corresponding to one $\mu$ cell) were identified in each 
waveform and their characteristics like amplitude, charge $\mathrm{Q}$ and arrival time have been determined. The device gain $\mathrm{G}$ has been calculated as $G=<Q>/ e$, where $\langle Q\rangle$ is the mean value of a single avalanche charge, e - is the elementary charge (more details on AC analysis can be found in the Refs. [6, 7]).

The gain of the device grows linearly with the bias voltage for all measured devices. The linear fit is used to determine the breakdown voltage $V_{B D}$ and $\mu$ cell capacitance $C_{\mu c e l l}$ (the slope of the line). Detailed analysis of $V_{B D}$ dependence on temperature for all SiPM samples as well as their comparison with $V_{B D}$ values determined from the IV characteristics are presented in the Section V (i.e. Fig. 3).

Laser light intensity was kept constant throughout the entire exposure but the average number of detected photons varies with the conditions, reflecting the variation of the Geiger probability with the operating conditions. Variation of the magnitude of the observed response to the laser (average number of detected photons, $\left.k_{\text {laser }}\right)$ can be used to determine the variation of the $P_{\text {Geiger. }}$ A value of $k_{\text {laser }}$ is determined by using the Poisson statistics:

$$
k_{\text {laser }}=-\ln (P(0))
$$

where $\mathrm{P}(0)$ is a fraction of recorded waveforms with no SiPM signal observed within a 20 ns gate centered on the expected arrival time of the laser signal. The observed value of $k_{\text {laser }}$ was corrected for dark SiPM pulses which can appear during the 20 ns laser gate using an auxiliary "dark" gate :

$$
k_{\text {laser }}^{\text {corr }}=-\ln (P(0))+\ln \left(P_{\text {dark }}(0)\right)=N_{\text {phot }} \times P_{\text {Geiger }}
$$

The absolute scale of the $P_{\text {Geiger }}$ is not known as we do not know the average number of photons illuminating the SiPM, $N_{\text {phot }}$. We have normalized the $P_{\text {Geiger }}$ to be 1 at the highest bias voltage at $T=+35^{0} \mathrm{C}$ (independently for each device). An example of the dependence of $P_{G e i g e r}$ on the overvoltage, $\Delta V$ $=V_{\text {bias }}-V_{B D}$, for measured SiPM samples is presented in Fig. 11. Following 


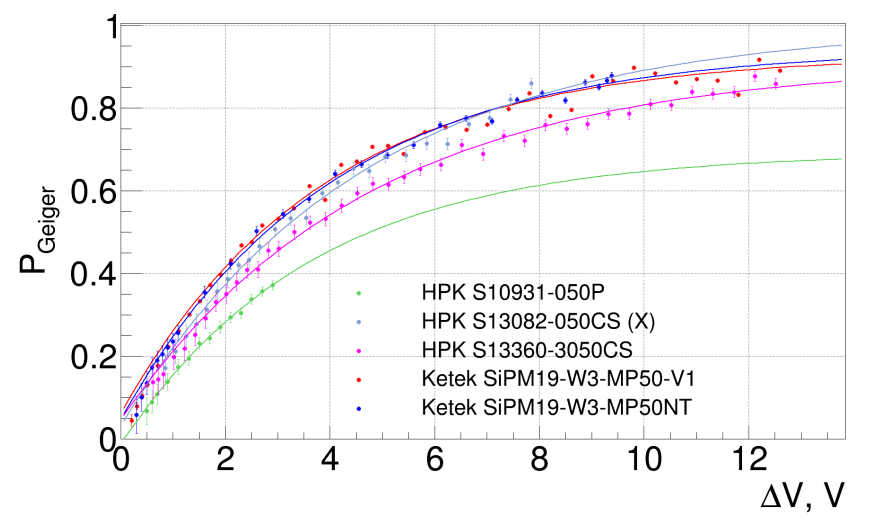

Figure 1: $P_{G e i g e r-A C}$ vs. $\Delta V$ at $25^{0} C$ for various SiPM samples

McIntyre [11] the $P_{\text {Geiger }}$ can be parameterized as:

$$
P_{\text {Geiger }}(\Delta V)=1-\exp (p \cdot \Delta V)
$$

where $\mathrm{p}$ is the parameter depending on the SiPM design and composition of free carriers. Such a parameterization provides a good description of our experimental data as shown in Fig. 1 (dots). Fig. 1 demonstrates that the working range of the 2011 HPK device was too small to allow reaching the maximum of $P_{\text {Geiger }}$, while the technology of 2015 devices, independent of the vendor, is greatly improved, allowing operation near the plateau of $P_{\text {Geiger }}$.

\section{Static DC acquisitions / IV model}

The experimental IV characteristics of SiPM samples from various vendors are presented in Fig. 2 for the temperature range $-35^{0} \mathrm{C}<T<+35^{0} \mathrm{C}$. For all temperatures and devices the IV curves have similar shapes indicating several regimes of the bias voltage:

- Pre-breakdown, $I_{p r e-B D}$, with relatively slow increase of the current being a combination of surface dark current (i.e. ionic impurities deposited on the surface during device fabrication) and the bulk dark current (i.e. 
Shockley-Read-Hall (SRH) thermal carrier's generated in the depleted region and carriers resulting from trap-assisted or band-to-band tunneling). We parameterize this component as:

$$
I_{\text {pre-BD }}\left(V_{\text {bias }}\right)=\exp \left(a \cdot V_{\text {bias }}+b\right)
$$

This parameterization ignores the increase of the carrier-induced current due to increase of the gain.

- "just-above" the breakdown voltage the current is dominated by the Geiger discharges produced by bulk free carriers. The current grows linearly with the overvoltage, it is in addition multiplied by the Geiger probability, which is the primary factor determining the shape of the IV curve in this region:

$$
I_{\text {post-BD }}\left(V_{\text {bias }}\right)=\frac{d N_{\text {carriers }}}{d t} \cdot C_{\mu c e l l} \cdot P_{\text {Geiger }} \cdot\left(V_{\text {bias }}-V_{B D}\right)^{2}
$$

- "far-above" the breakdown voltage the free carriers induced current is further amplified by afterpulsing and cross-talk effects. Due to their nonlinear nature the afterpulses are expected to be the dominant effect leading to the multiplicative factor 12 ]

$$
G_{A P}\left(V_{\text {bias }}\right)=1+\frac{P_{a p}\left(V_{\text {bias }}\right)}{1-P_{a p}\left(V_{\text {bias }}\right)}
$$

where $P_{a p}\left(V_{\text {bias }}\right)$ denotes the probability that an afterpulse will be produced by a single avalanche. $P_{a p}\left(V_{\text {bias }}\right)$ should be linearly rising with overvoltage and the recursive nature of afterpulsing leads to a continuous current at a some voltage, $V_{c r}$, where $P_{a p}\left(V_{c r}\right)=1$, thus

$$
P_{a p}\left(V_{\text {bias }}\right)=\frac{\Delta V}{V_{c r}-V_{B D}}
$$

- Post - "second breakdown" region, where run-away afterpulses and nonquenching effects lead to a continuous current limited by the total series resistance of the device. 

as:

$$
\begin{array}{r}
I_{\text {post-BD }}\left(V_{\text {bias }}\right)=\exp \left(a \cdot V_{\text {bias }}+b\right)+\frac{d N_{\text {carriers }}}{d t} \cdot \frac{V_{c r}-V_{B D}}{V_{c r}-V_{\text {bias }}} \times \\
\times\left(1-\exp \left[p \cdot\left(V_{\text {bias }}-V_{B D}\right)\right]\right) \cdot C_{\mu c e l l} \times\left(V_{\text {bias }}-V_{B D}\right)
\end{array}
$$

where a, b, $d N_{\text {carriers }} / d t, V_{c r}, V_{B D}, C_{\mu c e l l}$, and p are free parameters. This formula has been used to fit the experimental IV characteristics and the fits are shown in Fig. 2 a) to e) (i.e. lines). The values of $V_{B D}$ and $V_{c r}$ obtained from the fit are also indicated in the tables of Fig. 2

A very good agreement of the simple model (i.e. eq. 8) with the experimental data for all devices over large range of currents $10^{-12} A<I_{\text {post-BD }}<10^{-5} \mathrm{~A}$ allows for a robust determination of characteristics of SiPMs from the IV curves, in particular the breakdown voltage and the Geiger probability.

In the case of the $2011 \mathrm{HPK}$ device, the $V_{c r} \sim 2.7 \mathrm{~V}$ and our model describes well the experimental data over the entire working range showing that the physical phenomena determining the $2^{n d}$ breakdown of this device is the afterpulsing, as described by the model. Discrepancy between the model and the data just below the breakdown voltage, especially at higher temperatures, is attributed to the neglect of the contribution of proportional amplification.

Recent technological improvements have resulted in major reduction of afterpulsing and cross-talk thus allowing a much larger range of the bias voltages. Comparing the model with the IV curves for recent devices, we observe that at large overvoltage values there are indications of additional physical phenomena 165 which contribute to an additional increase of the current (i.e. internal heating, non-quenched pulses) and may limit the range of applicability of the simple model presented here. The overvoltage region and the magnitude of these new phenomena depends on the vendor, hence it may be related to the detector design or to technological details. 


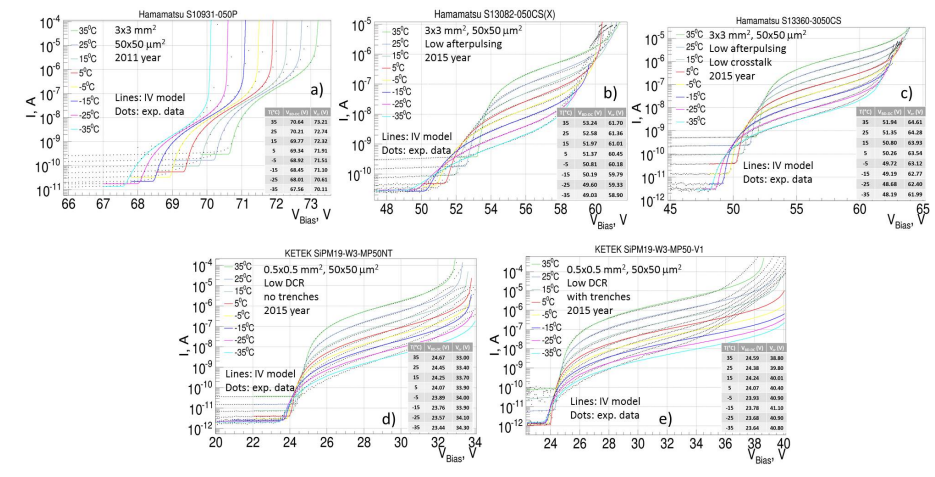

Figure 2: Reverse IV characteristics (dotes) and fit (lines) for various SiPM samples in T range $-35^{\circ} \mathrm{C}<T<+35^{0} \mathrm{C}$

\section{Comparison of parameters from DC / AC acquisitions}

A comparison between $V_{B D-D C}$ values obtained from DC acquisitions /IV model and the $V_{B D-A C}$ calculated from $\mathrm{AC}$ acquisitions/ waveform analysis at various temperatures is presented in Fig. 3. Both methods are in good agreement regarding the temperature variation of the breakdown voltage, but there 175 is a significant and systematic difference: the values of the $V_{B D-D C}$ are few hundreds $\mathrm{mV}$ higher than the values of $V_{B D-A C}$ for all measured devices and temperatures. This discrepancy reflects the fundamental difference in the physical significance of the "breakdown" voltage determined by these two methods. The "IV-based" measurement is sensitive to the onset of avalanche phenomenon and it determines the "breakdown" as defined by the fundamental papers of McIntyre. The AC/gain linearity method determines the voltage to which the voltage across the diode is reduced to quench the avalanche. The latter is naturally lower than the former and our results are consistent with this expectation.

Fig. 4 shows a comparison between $P_{\text {Geiger-DC }}$ shape obtained from DC acquisitions /IV model and the $P_{\text {Geiger-AC }}$ shape calculated from AC acquisitions/ waveform analysis at various temperatures for Hamamatsu S133603050CS device. A very good agreement is observed between two methods for $-35^{\circ} \mathrm{C}<\mathrm{T}<+15^{0} \mathrm{C}$, while an emerging disagreement is observed at higher 

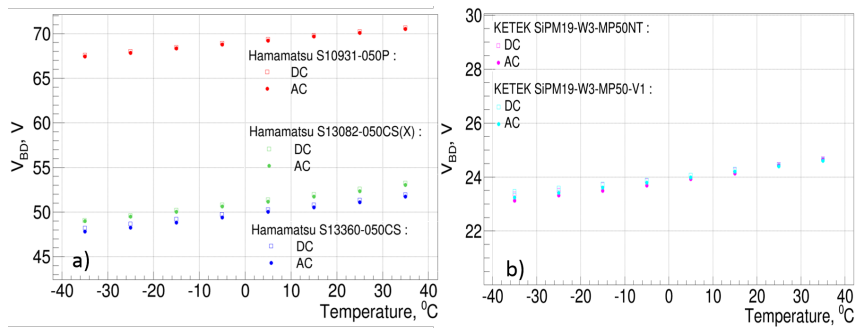

Figure 3: $V_{B D-D C}$ and $V_{B D-A C}$ vs temperature for various SiPMs: a) HPK and b) KETEK
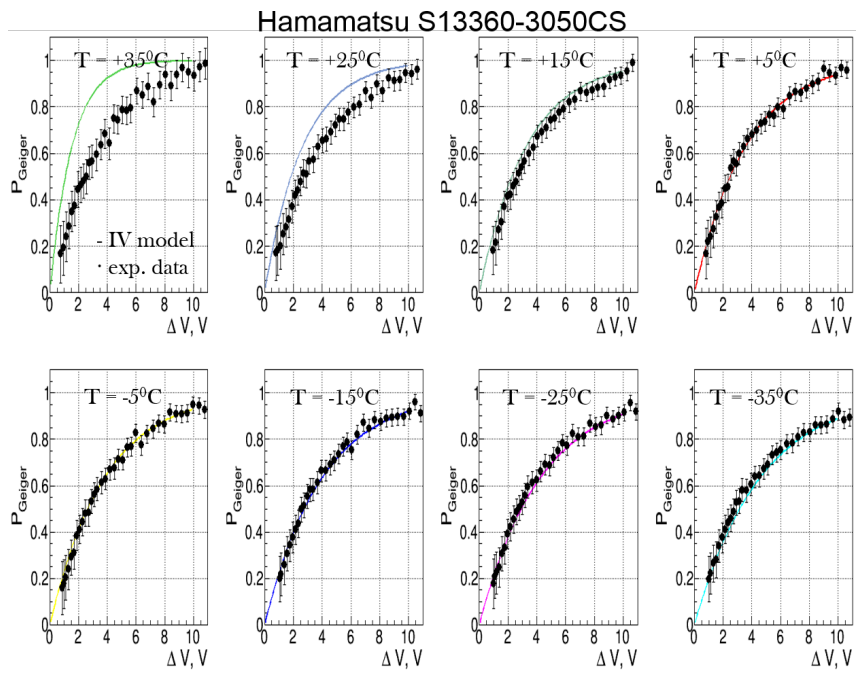

Figure 4: $P_{\text {Geiger }-D C}$ and $P_{\text {Geiger }-A C}$ vs. $\Delta V$ of an HPK SiPM (2015) at various T

temperatures, $25^{0} \mathrm{C}$ and above. These studies are in their preliminary stages

190

IV curve is due to avalanches produced by free carriers (presumably generated by both electrons and holes) whereas $\mathrm{AC}$ acquisition is performed with red light (i.e. avalanches are mostly generated by holes since our devices are $p^{+} / n / n-e p i / n-t y p e$ design). Therefore, the difference between the two Geiger 195 probabilities at high temperatures could be related to the difference in the nature of the primary carriers [13]. 


\section{Conclusions}

In this paper we reported a physical model describing the SiPM IV shape over full device working range. Devices from two vendors and different production runs were studied in a temperature range of $-35^{0} \mathrm{C}<T<+35^{0} \mathrm{C}$. It has been shown that the shape of the IV curve can be described in terms of the Geiger probability and afterpulsing for all devices in the bias range of $V_{B D}<V_{\text {bias }}<$ $V_{c r}$ and large currents range of $10^{-12} A<I_{\text {post-BD }}<10^{-5} A$. The model allows determination of important device parameters as breakdown voltage $V_{B D}$ and the shape of triggering probability $P_{G e i g e r}$. The $V_{B D}$ is slightly higher (few hundreds $\mathrm{mV}$ ) than the "breakdown voltage" determined from the usual method of a linear fit of gain as a function of bias voltage. The difference could be related to the different physical interpretation of the resulting parameters. The recent generation of SiPMs have a very wide working range and there is an evidence phenomena beyond afterpulsing like internal heating or non-quenched pulses contributing to the current at higher bias voltage.

\section{Acknowledgment}

The authors thanks to LAL mechanical and infrastructure services for the precious help in designing and building the cryogenic set-up, as well as to SERDI/GRED group for providing lasers and measurement instruments. A special thanks is given to Hammoudi Nourredine from IPNO laboratory for his advises on calibration of the cryogenic set-up. This work was supported by the Laboratory of Linear Accelerator (LAL) and the Labex Physique des 2 Infinis et des Origines (P2IO), under the project SONIM. Fermi National Accelerator Laboratory is operated by Fermi Research Alliance, LLC under Contract No. De-AC02-07CH11359 with the United States Department of Energy.

\section{References}

[1] G. Llosa, N. Belcari, M. Bisogni, G. Collazuol, S. Marcatili, M. Boscardin, M. Melchiorri, A. Tarolli, C. Piemonte, N. Zorzi, P. Barrillon, S. Bondil- 
Blin, V. Chaumat, C. de La Taille, N. Dinu, V. Puill, J.-F. Vagnucci, A. D. Guerra, First results in the application of silicon photomultiplier matrices to small animal PET, Nuclear Instruments and Methods in Physics Research Section A: Accelerators, Spectrometers, Detectors and Associated Equipment 610 (2009) 196 - 199. New Developments In Photodetection NDIP08.

[2] N. Dinu, T. A. Imando, A. Nagai, L. Pinot, V. Puill, S. Callier, B. Janvier, C. Esnault, M.-A. Verdier, L. Raux, V. Vandenbussche, Y. Charon, L. Menard, SiPM arrays and miniaturized readout electronics for compact gamma camera, Nuclear Instruments and Methods in Physics Research Section A: Accelerators, Spectrometers, Detectors and Associated Equipment 787 (2015) 367 - 372. New Developments in Photodetection NDIP14.

[3] D. Jeans, the CALICE collaboration, Results of the CALICE scintillator ECAL beamtest at DESY, Journal of Physics: Conference Series 160 (2009) 012026 .

[4] L. Gruber, G. S. M. Ahmed, S. E. Brunner, P. Bhler, J. Marton, K. Suzuki, Position sensitive SiPM detector for Cherenkov applications, Journal of Instrumentation 6 (2011) C11024.

[5] S. Korpar, Status and perspectives of solid state photon detectors, Nuclear Instruments and Methods in Physics Research Section A: Accelerators, Spectrometers, Detectors and Associated Equipment 639 (2011) 88 93. Proceedings of the Seventh International Workshop on Ring Imaging Cherenkov Detectors.

[6] A. Nagai, Silicon Photomultiplier for Medical Imaging Analysis of SiPM characteristics-, Proceedings of Journes de Rencontre Jeunes Chercheurs (2013) 43-47.

[7] N. Dinu, A. Nagai, A. Para, Studies of MPPC detectors down to cryogenic temperatures, Nuclear Instruments and Methods in Physics Research Sec- 
tion A: Accelerators, Spectrometers, Detectors and Associated Equipment 787 (2015) 275 - 279. New Developments in Photodetection \{NDIP14\}.

[8] N. Serra, G. Giacomini, A. Piazza, C. Piemonte, A. Tarolli, N. Zorzi, Experimental and TCAD Study of Breakdown Voltage Temperature Behavior in $n^{+} / p$ SiPMs, IEEE Transactions on Nuclear Science 58 (2011) 12331240.

[9] E. Garutti, M. Ramilli, C. Xu, W. Hellweg, R. Klanner, Characterization and X-ray damage of Silicon Photomultipliers, Proceedings of Science PoS(TIPP2014)070 (2014).

[10] N. Dinu, C. Bazin, V. Chaumat, C. Cheikali, A. Para, V. Puill, C. Sylvia, J. F. Vagnucci, Temperature and bias voltage dependence of the MPPC detectors (2010) 215-219.

[11] R. J. McIntyre, Theory of Microplasma Instability in Silicon, Journal of Applied Physics 32 (1961) 983-995.

[12] A. Para, Afterpulsing in Silicon Photomultipliers: Impact on the Photodetectors Characterization, arXiv 1503.01525 [physics.ins-det] (2015).

[13] W. G. Oldham, R. R. Samuelson, P. Antognetti, Triggering phenomena in avalanche diodes, IEEE Transactions on Electron Devices 19 (1972) 1056-1060. 